\title{
MiR-128 and miR-125 regulate expression of Coagulation Factor IX gene with nonsense mutation by repressing nonsense-mediated mRNA decay
}

\author{
Gang Wang ${ }^{a}$, Baofeng Chai ${ }^{\text {b,* }}$, Linhua Yang ${ }^{a, * *}$ \\ ${ }^{a}$ Department of Hematology, The Second Clinical Medical College, Shanxi Medical University, \\ Taiyuan 030001, PR China \\ ${ }^{\mathrm{b}}$ Key Laboratory of Chemical Biology and Molecular Engineering of Ministry of Education, \\ Institute of Biotechnology, Shanxi University, Taiyuan 030006, PR China \\ * Corresponding author: Tel.: +86 3517019083. \\ *** Corresponding author: Tel.: +86 3513365282 . \\ E-mail: bfchai@sxu.edu.cn (B. Chai), yanglh5282@163.com (L. Yang)
}


ABSTRACT: Hemophilia could be caused by a nonsense mutation of the Factor IX gene, leading to a deficiency of Factor IX (F9). The nonsense mutation frequency of F9 is more than $10 \%$ according to the database. Nonsense-mediated mRNA decay (NMD) is a defined cellular response that can potentially prevent the production of such deleterious C-terminal truncated proteins from aberrant mRNA. Here, we constructed a mini-gene of Factor IX (Mini-hF9) and some nonsense mutants and characterized the mini-gene splicing pattern. We discovered that NMD regulated mini-hF9 expression in two nonsense mutants: E7a (nt $34 \mathrm{G}>\mathrm{T}$ in Exon 7 ) and E7b (nt $52 \mathrm{G}>\mathrm{T}$ in Exon 7), but not in another nonsense mutants: E7c (nt $85 \mathrm{G}>\mathrm{T}$ in Exon 7) and E8 (nt $42 \mathrm{C}>\mathrm{T}$ in Exon 8). In addition, mini-hF9 transcripts were accumulated after transfection with miR-128 or miR-125 mimics in E7a and E7b. Our results suggest that PTC (premature termination codon) location is a key determination for triggering NMD; miR-128 and miR-125 could help to increase the nonsense-mutant F9 levels by repressing NMD.

KEY WORDS: microRNA; hemophilia; Factor IX; nonsense mutation; NMD

\section{Introduction}

The coagulation factor IX (F9) gene encodes a serine protease that is critical for blood clotting and coagulation. Individuals with severe hemophilia B have functional F9 levels that are less than $1 \%$ of normal values and have frequent bleeding events, which are commonly associated with crippling arthropathy and early death [1,2]. Restoration to $\sim 5 \%$ activity converts severe hemophilia B to a mild form [3]. There are 87 nonsense mutations in all 799 point mutations, according to the Hemophilia B Mutation Database (http://www.factorix.org/). 
Transcripts containing premature termination codons (PTCs) are a serious problem for cells, as they may encode C-terminal truncated proteins with dominant-negative or deleterious gain-of-function activity [4,5]. Nonsense-mediated decay (NMD), a pathway that degrades mRNAs containing nonsense codons, has been defined that can potentially prevent the production of such deleterious C-terminal truncated proteins from PTC-containing mRNA. In eukaryotic cells, NMD requires both active mRNA translation and NMD-specific trans-acting factors [6]. Phosphorylation/dephosphorylation cycles of UPF1 are essential for NMD [7]. The SMG1 protein mediates the phosphorylation of UPF1, and the SMG5, SMG6 and SMG7 proteins mediate the dephosphorylation of UPF1 [7,8,9]. Mammalian tissue culture studies have established the splicing-dependent exon-exon junction complex (EJC) as another important trans-acting component for NMD $[8,10,11]$. In mammals, NMD cannot distinguish PTCs in the penultimate exons that are located less than $\sim 55$ base pairs (bp) from the final intron [4].

MicroRNAs (miRNAs) are small ( $\sim 23 \mathrm{nt})$, regulatory, noncoding RNA molecules that control the expression of their target mRNAs predominantly by binding to the 3' untranslated region (UTR) [12,13]. MiR-128 was recently reported to repress NMD function by targeting UPF1 and the EJC core component MLN51 (also known as CASC3 or BTZ) in mammals [14]. Specifically, induction of the brain (and thymus) -restricted miRNA-128 early in brain development reduces NMD efficiency by targeting mRNAs encoding UPF1 and MLN51, thereby reducing the functional levels of these proteins $[15,16]$. Another miRNA that may contribute to this regulation is miR-125 (including miR-125a and miR-125b), a neurally expressed miRNA that recently shown to repress the expression of another NMD factor SMG1 $[16,17]$. These data suggest the existence of a conserved mRNA circuit that links the microRNA and NMD pathways and results in the 
induction of cell type-specific transcripts during development.

In this work, we constructed a mini-gene of Factor IX (Mini-hF9) and some nonsense mutants and characterized the mini-gene splicing pattern. In two nonsense mutants, E7a (nt $34 \mathrm{G}>\mathrm{T}$ in Exon 7) and E7b (nt $52 \mathrm{G}>\mathrm{T}$ in Exon 7), transcripts were observed to be regulated by NMD, which had a premature termination codon (PTC) located $>55$ nucleotides upstream of the last exon-exon junction. In another mutants, E7c (nt $85 \mathrm{G}>\mathrm{T}$ in Exon 7) and $\mathrm{E} 8$ (nt $42 \mathrm{C}>\mathrm{T}$ in Exon 8), the levels of F9 transcripts did not decrease compared with wild type, which had a PTC located less than 55 nucleotides upstream of the last exon-exon junction or in the last exon. In addition, we discovered that mini-hF9 transcripts were accumulated after transfection with miR-128 or miR-125 mimics in E7a and E7b. Our results suggest that PTC (premature termination codon) location is a key determination for triggering NMD; miR-128 and miR-125 could help to increase the nonsense-mutant F9 levels by repressing NMD.

\section{Materials and Methods}

\subsection{Construction of the mini gene and nonsense mutants}

A mini gene of Factor IX (Mini-hF9) was cloned into the pcDNA3.1 (-) vector. $6 \times \mathrm{Myc}$ and $6 \times$ HA tags were added at the 5'- and 3'- ends of the mini gene, respectively, to analyze the expression of the mini gene. The Mini-hF9 gene contains three parts: the first part contains the whole exon 1 and the 5' partial intron 1; the second part contains the $3^{\prime}$ partial intron 5 , the whole exon 6 and the 5' partial intron 6; and the last part contains the 3 ' partial intron 6 , the whole exon 7 , 
intron 7, and exon 8 followed by termination codon (Figure 1A). We then obtained the nonsense mutants E7a (nt $34 \mathrm{G}>\mathrm{T}$ in Exon 7), E7b (nt $52 \mathrm{G}>\mathrm{T}$ in Exon 7), E7c (nt $85 \mathrm{G}>\mathrm{T}$ in Exon 7) and E8 (nt $42 \mathrm{C}>\mathrm{T}$ in Exon 8) using PCR mutagenesis to analyze the mechanism of PTC recognition (Figure 1B).

\subsection{Cell culture, transfection and RNAi}

HeLa, HEK293T, COS-7 and HepG2 cells were cultured in DMEM (HyClone) supplemented with $10 \%$ fetal calf serum (HyClone) and grown at $37^{\circ} \mathrm{C}$ in a humidified atmosphere of $5 \% \mathrm{CO}_{2}$ and $95 \%$ air, according to standard procedures.

Transfections were performed with the Attractene Transfection Reagent (Qiagen) in 12-well or 24-well plates with either plasmids and/or microRNA mimics, according to the manufacturer's instructions.

RNAi was performed using the RNAi-Ready pSIREN-DNR-DsRed-Express Donor Vector (TAKARA) containing shRNA driven by the U6 promotor. Cells were harvested 24 or 48 hours after transfection for RNA or protein extractions. shRNA target sequences $[18,19]$ are listed in Table 1.

\subsection{Analysis of Mini-hF9 splicing pattern}

Total RNA was isolated respectively from HeLa, HEK293T, COS-7 and HepG2 cells transfected with the Mini-hF9 expression vector using the RNAiso plus (TAKARA). 
Oligo dT primers, random primers and PrimerScript ${ }^{\circledR}$ RT Master Mix (TAKARA) were used to perform first-strand complementary DNA (cDNA) synthesis from $500 \mathrm{ng}$ of total RNA, according to the manufacturer's protocol. Of a total of $10 \mu \mathrm{L}, 0.5 \mu \mathrm{L}$ was used as a template for the PCR reactions with gene-specific primers. The PCR primers are BF319 and BF320 (primer sequences are listed in Table 1). PCRs were carried out under standard conditions using Easy $\mathrm{Taq}^{\mathrm{TM}}$ DNA polymerase (TransGen Biotech). The PCR products were sequenced by the TAKARA Corporation.

\subsection{NMD analysis}

HeLa cells were cultured and transfected with the Mini-hF9 or nonsense mutant expression vector as described above. 46 hours after transfection, cells were treated for 2 hours with cycloheximide (100 $\mu \mathrm{g} / \mathrm{mL}$; Sigma), a drug that can inhibit NMD by prematurely terminating translation.

After treatment with this drug, the cells were washed twice with phosphate-buffered saline (PBS). Total RNA was isolated using an RNAiso Plus (TAKARA) according to the manufacturer's protocol, and $500 \mathrm{ng}$ of the RNA was used for reverse transcription using PrimerScript ${ }^{\circledR}$ RT Master Mix (TAKARA). Quantitative RT-PCR (qPCR) assays included $0.4 \mu \mathrm{M}$ aliquots of each primer and 12.5 ng cDNA in SYBR Premix Ex Taq ${ }^{\mathrm{TM}}$ II Tli RnaseH Plus (TAKARA). PCR reactions were performed on an ABI StepOne Plus (Applied Biosystems). Quantification of gene expression was based on the $\Delta \Delta \mathrm{CT}$ method and normalized to neomycin (Neo) gene levels. A melting curve was constructed and an electrophoresis analysis was performed to control PCR 
product specificities and exclude nonspecific amplification. The PCR primers for Mini-hF9 mRNA are BF321 and BF324 (primer sequences are in Table 1). The PCR primers for Neo are BF325 and BF326 (primer sequences are listed in Table 1).

To further confirm our results, HeLa cells were cultured and co-transfected with RNAi expression vectors as described above. 48 hours after co-transfection, total RNA was extracted and used for reverse transcription. The mRNA levels were detected using qPCR assays as described above.

\subsection{Protein extractions and western blotting analysis}

COS-7 cells and HEK293T cells were cultured and transfected with the Mini-hF9 or nonsense mutant expression vector as described above. 48 hours after transfection, the cells were washed in cold PBS, and protein was extracted using the cell lysis buffer $(20 \mathrm{mM}$ Tris $\mathrm{pH} 7.5,150 \mathrm{mM} \mathrm{NaCl}$, 1\% Triton X-100; Beyotime), according to the manufacturer's instructions.

The protein extracts were boiled, resolved by 15\% SDS-PAGE and electroblotted onto $0.45-\mu \mathrm{m}$ pore-size nitrocellulose membranes (Millipore) for 1 hour at $90 \mathrm{~V}$ using a Tran-Blot Electrophoretic Transfer Cell (Bio-Rad). The membranes were soaked in blocking buffer (5\% skim milk, $140 \mathrm{mM} \mathrm{NaCl}, 27 \mathrm{mM} \mathrm{KCl}, 10 \mathrm{mM} \mathrm{Na} 2 \mathrm{HPO}_{4}, 1.8 \mathrm{mM} \mathrm{KH}_{2} \mathrm{PO}_{4}, 0.5 \%$ Tween-20) for 1 hour at room temperature and incubated at room temperature for 1 hour with a mouse monoclonal anti-myc antibody (catalog no. M4439; Sigma-Aldrich) diluted 1:2000 or anti-HA antibody (catalog no.HT301; TransGen Biotech) diluted 1:2000 in blocking buffer. The membranes were washed three times with wash buffer $(140 \mathrm{mM} \mathrm{NaCl}, 27 \mathrm{mM} \mathrm{KCl}, 10 \mathrm{mM}$ 
$\mathrm{Na}_{2} \mathrm{HPO}_{4}, 1.8 \mathrm{mM} \mathrm{KH} \mathrm{PO}_{4}, 0.5 \%$ Tween-20) for 5 minutes each wash and incubated at room temperature for 1 hour with an HRP-goat anti-mouse $\operatorname{IgG}$ (catalog no. 81-6520; Invitrogen) diluted 1:10000 in blocking buffer. Proteins were detected using a highly sensitive enhanced chemiluminescent substrate (SuperSignal West Dura Extended Duration Substrate; Pierce Chemical). Western blotting gray scale results were used to semiquantitative analysis.

Similarly, membranes were incubated at room temperature for 1 hour with mouse monoclonal anti-hUPF1 antibody (catalog no. Sc-166091; Santa Cruz) or mouse monoclonal anti-hSMG1 antibody (catalog no. Sc-135563; Santa Cruz) diluted 1:200 in blocking buffer. Secondary antibody dilution was as described above. $\beta$-actin is the internal control. Rabbit anti- $\beta$-actin (catalog no. bs-0061R; Biosynthesis) was diluted 1:1000, and HRP-goat anti-rabbit Ig G (catalog no. 81-6120; Invitrogen) was diluted 1:50000 in blocking solution at room temperature for 1 hour. Proteins were detected as described above.

\subsection{Statistical Analysis}

Student's two-sided t-test was used to compare values of test and control samples. $\mathrm{P}<0.05$ was considered to be statistically significant.

\section{Results}

\subsection{Expression of the Mini-hF9 gene}

Total RNA was isolated from HeLa cells transfected with the Mini-hF9 or nonsense mutant expression vectors. RT-PCR primers were designed to cover the myc and HA tags (Figure 1A, 
shown with arrows). In the RT-PCR assay, no altered splicing events were detected in HeLa, HEK293 T, COS-7 and HepG2 cells transfected with the Mini-hF9 gene expression vector (Figure 1C). The RT-PCR products were sequenced with the reverse primer. The results confirmed the analyzed splicing event (shown in Figure 1A). In the Western Blot assay, the only full length protein was detected in cells transfected with the Mini-hF9 gene expression vector (Figure 1D). According to the analysis, we demonstrated that the normal transcripts and full length proteins were expressed in cells transfected with the Mini-hF9 gene expression vector.

\subsection{NMD occurs in E7a and E7b mutants}

To identify mutants in which NMD might occur, we measured the levels of the Mini-hF9 mRNA in HeLa cells transfected with the Mini-hF9 or nonsense mutant expression vector for 24 or 48 hours (Figure 2A). The q-PCR results showed that the levels of the Mini-hF9 mRNA decreased to half in E7a and E7b mutants 24 hours after transfection. It decreased greatly to almost 20\% 48 hours after transfection. In contrast, the levels of Mini-hF9 mRNA did not increase or decrease significantly in E7c and E8 mutants. Similar results also occurred in HEK293T and HepG2 (data not shown). We concluded that NMD could occur in the two nonsense mutants: E7a and E7b, but not in others.

To confirm it, Mini-hF9 mRNA levels were detected with $100 \mu \mathrm{g} / \mathrm{mL}$ cycloheximide (CHX) treatment (Figure 2B) or with depletion of the NMD proteins hUPF1 and hSMG1 (Figure 2C and 2D). We used the RNAi technique to decrease hUPF1 and hSMG1 levels. q-PCR results showed 
that the level of Mini-hF9 mRNA increased significantly after CHX, RNAi-hUPF1 or RNAi-hSMG1 treatment. Western blot analysis indicated that the antisense hUPF1 expression vector can decrease UPF1 protein levels (Figure 2E); similarly, the antisense hSMG1 expression vector could decrease SMG1 protein levels (Figure 2F). Therefore, we believed that NMD degraded PTC-containing transcripts in E7a and E7b mutants but not in others. PTC location is a key determinant for triggering NMD.

\subsection{Western blotting assay of Mini-hF9 protein}

After determining the mRNA levels in the Mini-hF9 and nonsense mutants, we detected the levels of the full-length and C-terminal truncated proteins using an anti-myc antibody. Western blotting analysis performed on lysates of HEK293T cells transfected with the Mini-hF9 or nonsense mutant expression vector showed different bands with different molecular weights ranging from $20 \mathrm{kD}$ to $80 \mathrm{kD}$ (Figure $3 \mathrm{~A}$ ).

The full-length proteins were detected in the lysates of cells transfected with the Mini-hF9 expression vector. A band corresponding to a molecular weight of approximately $60 \mathrm{kD}$ was observed. The slight band in the wild type is probably due to the high molecular weight of the protein, which leads to the low transfer efficiency. The bands were detected in other samples with a lower molecular weight of approximately $25 \mathrm{kD}$. The bands were faint in the lysates of cells transfected with E7a and E7c (Figure 3A and 3B), and it confirmed that NMD occurred in the two nonsense mutants 


\section{4 miR-128 and miR-125 regulate Mini-hF9 expression in E7a and E7b mutants}

Recently, it was reported that miR-128 [14] and miR-125 [17] could repress the NMD pathway by targeting UPF1, EJC core component NLN51 or SMG1 in mammals. So we concluded that miR-128 or miR-125 (including miR-125a and miR-125b) could enhance the stability of Mini-hF9 mRNA in E7a or E7b by preventing NMD. To confirm it, Mini-hF9 mRNA levels were detected in HeLa cells co-transfected with E7a expression vector and miR-128 mimics (miR-125 or control mimics) (Figure 4A). The q-PCR results showed that the levels of the Mini-hF9 mRNA increased to more than twice in E7a mutant after miR-128 or miR-125 mimics treatment. Similar results also occurred in E7b mutant (Figure 4B). Therefore, we believed that miR-128 and miR-125 could improve the stability of Mini-hF9 mRNA in E7a and E7b mutants by NMD inhibition.

\section{Discussion}

Gene expression comprises a series of interconnected steps, including transcription, mRNA maturation, translation, and mRNA degradation. Naturally, diverse regulatory mechanisms have evolved to ensure the accuracy of gene expression at multiple levels [8]. One of these mechanisms is the nonsense-mediated mRNA decay (NMD) pathway, which degrades mRNAs containing premature termination codons (PTC).

In mammals, PTCs in the penultimate exons that are located less than $\sim 55$ bp from the final 
exon-exon junction fail to elicit efficient NMD. This result is believed to reflect the location of the EJC (exon-exon junction complex) on spliced mRNAs; a translocating ribosome would displace the EJC upstream of the last exon-exon junction before the ribosome could recognize the termination codon located less than $\sim 55 \mathrm{bp}$ from the exon-exon junction [20]. In our constructed nonsense mutant E7c (nt $85 \mathrm{G}>\mathrm{T}$ in Exon 7) mRNA, a PTC was located 30 bp upstream of the final exon-exon junction (Figure 1B). In another mutant, E8 (nt $42 \mathrm{C}>\mathrm{T}$ in Exon 8), the PTC was located in the last exon. The results showed that NMD could not be elicited. However, there are several genes whose mRNA degradation appears to show exceptions to this rule, including hexoseaminidase A (HEXA) [21], MPZ (encoding myelin protein zero) [22], HNF-1 (hepatocyte nuclear factor-1 $\beta$ ) [23], and the T-cell receptor (TCR) [24] genes of the cellular immune system [4]. The failure of these genes to follow the ' $\sim 55$ bp boundary rule' suggests that alternative or additional signals might elicit NMD. Studies have shown that certain cis-acting elements, such as long 3' UTRs, are sufficient to elicit NMD [8]. Nevertheless, neither a downstream EJC nor a long 3' UTR is absolutely required for NMD. The only requirement for NMD is translation-dependent recruitment of UPF1 to the mRNA.

The NMD pathway can potentially prevent the production of such deleterious C-terminal truncated proteins from PTC-containing mRNAs. However, there are numerous, well-studied examples of human diseases resulting from nonsense or frameshift mutations $[4,8,25]$. Up to $30 \%$ of mutant alleles that contribute to human diseases harbor a premature translation termination signal through either nonsense or frameshift mutations [26,27]. Recently, many genetic disorders have been analyzed to detect NMD triggering mutations because treatment strategies might be easier to design compared to other types of mutations [28]. However, there are no effective 
treatments for many diseases caused by PTC-containing mutations. Remarkably, only $1-5 \%$ of normal protein levels, according to PTC read-through approaches, can significantly eliminate or reduce the principal manifestations of PTC-associated disorders, such as cystic fibrosis and Hurler syndrome $[8,29]$. Therefore, it is important to clearly explain the molecular mechanism of diseases caused by nonsense mutation in order to cure these diseases.

Ataluren (formerly PTC124), a 284-Dalton 1,2,4-oxadiazole, has been shown to inhibit nonsense mutations by promoting ribosomal read-through of PTCs in transcripts and offer therapeutic potential for multiple nonsense mutation-mediated diseases.[30,31] This small molecular compound has a low toxicity and effectively acts on the PTCs, rather than all stop condons. So we would like to suppose that PTC124 treatment could increase the levels of mRNA targeted by NMD according to promoting read-through. However, our results were different from our prediction. The levels of the norm mRNA did not significantly changed after PTC124 treatment in nonsense mutants, E7a and E7b (data not shown). The experiment about read-through of PTC after PTC124 treatment is still in progress.

MicroRNAs (miRNAs) are short non-coding RNA molecules which have been considered to involve in the regulation of gene expression. miRNAs could target and regulate specific gene transcripts, so they have the potential as therapeutic drugs. We also confirmed miR-128 and miR-125 could increase the level of the Mini-hF9 mRNA in nonsense mutant E7a and E7b by NMD inhibition (Figure 4A and 4B). Thus, we propose a new treatment program for nonsense mutation-mediated diseases. Cells could be co-treated with miR-128 (or miR-125) and read-through drugs (PTC124), then the mRNA levels should increase and more full-length proteins should be translated. 


\section{Acknowledgments}

The authors thank Dr Guoqiang Zhang (Institute of Zoology, Chinese Academy of Sciences, Beijing, China) for helping in cell culture and transfection, Dr Zhiyun Zhang (Institute of Biotechnology, Shanxi University, Taiyuan, China) for providing RNAi expression vector. This work was supported by the National Science Foundation of China (No.31172078; 30770294), the Natural Science Foundation of Shanxi (2015011119), the Post Doctoral Fund of Shanxi medical university and the Doctoral Fund of the Second Hospital of Shanxi medical university.

\section{Figure legend}

Figure 1. Construction and expression of Mini-hF9 gene. (A) Diagram of the full-length Mini-hF9 precursor mRNA and normally spliced mRNAs derived from this mRNA. (B) Diagram of the PTC location in E7a, E7b, E7c and E8 mutants. (C) RT-PCR products obtained with the primer couple (BF319 and BF320, shown as arrows in Figure 1A) on a 1.2\% agarose gel. Lane Mr indicates molecular weight marker. Lane Ctrl indicates RT-PCR products from cells transfected with pcDNA3.1 (-) expression vectors. No altered splicing events were detected in cells transfected with the Mini-hF9 gene expression vector. The only RT-PCR products were found and confirmed by sequencing. (D) Western blotting analysis of myc/HA-tagged Mini-hF9 protein in COS-7 and HEK293T cells. Lane Ctrl indicates proteins from cells transfected with pcDNA3.1 (-) expression vectors. The only full length protein was detected in cells transfected with the Mini-hF9 gene expression vector. 
Figure 2. NMD occurred in E7a and E7b mutants. (A) q-PCR analysis of Mini-hF9 mRNA levels in HeLa cells transfected for 24 or 48 hours with Mini-hF9 or nonsense mutant expression vectors. Shown are the results from three independent experiments normalized to Neo mRNA levels. ${ }^{* *} \mathrm{P}$ $<0.01$ compared to the WT plasmid. (B) q-PCR analysis of Mini-hF9 mRNA levels in HeLa cells 48 hours after transfection as in (A). Quantification was conducted as in (A). Cycloheximide (CHX), which was added to a final concentration of $100 \mu \mathrm{g} / \mathrm{mL}$, was (CHX+) or was not (CHX-) added two hours prior to cell harvest. ** $\mathrm{P}<0.01$ compared to the cells treated without $\mathrm{CHX}$. (C and D) q-PCR analysis of HeLa cells co-transfected for 48 hours with Mini-hF9 (or nonsense mutants) and antisense-hUPF1 (or antisense-hSMG1) expression vector. Quantification was conducted as in $(\mathrm{A}) .{ }^{* *} \mathrm{P}<0.01$ compared to the cells transfected with a negative RNAi expression plasmid. (E and F) Western blotting analysis of endogenous UPF1 and SMG1 protein levels in HKE 293T cells 48 hours after transfection with antisense-hUPF1 or antisense-hSMG1 expression vector. $\beta$-actin is used as the internal control.

Figure 3. Western blotting analysis of myc-tagged Mini-hF9 protein levels in HEK293T cells. (A) Western blotting analysis of myc-tagged Mini-hF9 protein levels in HEK293T cells 48 hours after transfection with Mini-hF9 or nonsense mutant expression vectors. X-ray film was exposed for 2 minutes. $\beta$-actin is used as the internal control. (B) Western blot analysis was conducted as in (A). The exposure time was extended to 40 minutes to detect the protein level in E7a.

Figure 4. miR-128 and miR-125 regulate Mini-hF9 expression in E7a and E7b mutants. (A) q-PCR analysis of Mini-hF9 mRNA levels in HeLa cells transfected for 48 hours with E7a 
expression vector and miR-128 (miR-125a, miR-125b or control miRNA) mimics. Shown are the results from three independent experiments normalized to Neo mRNA levels. (B) q-PCR analysis of Mini-hF9 mRNA levels in HeLa cells transfected for 48 hours with E7b expression vector and miR-128 (miR-125a, miR-125b or control miRNA) mimics. Quantification was conducted as in (A). $* \mathrm{P}<0.05$ compared to the cells transfected with negative control miRNA mimics.

\section{References}

[1] A.C. Nathwani, E.G. Tuddenham, S. Rangarajan, et al., Adenovirus-associated virus vector-mediated gene transfer in hemophilia B, N Engl J Med 365 (2011) 2357-2365.

[2] S.C. Darby, S.W. Kan, R.J. Spooner, et al., Mortality rates, life expectancy, and causes of death in people with hemophilia A or B in the United Kingdom who were not infected with HIV, Blood 110 (2007) 815-825.

[3] H. Li, V. Haurigot, Y. Doyon, et al., In vivo genome editing restores haemostasis in a mouse model of haemophilia, Nature 475 (2011) 217-221.

[4] M. Khajavi, K. Inoue, J.R. Lupski, Nonsense-mediated mRNA decay modulates clinical outcome of genetic disease, Eur J Hum Genet 14 (2006) 1074-1081.

[5] Y.F. Chang, J.S. Imam, M.F. Wilkinson, The nonsense-mediated decay RNA surveillance pathway, Annu Rev Biochem 76 (2007) 51-74.

[6] S. Brogna, J. Wen, Nonsense-mediated mRNA decay (NMD) mechanisms, Nat Struct Mol Biol 16 (2009) 107-113.

[7] T. Ohnishi, A. Yamashita, I. Kashima, et al., Phosphorylation of hUPF1 induces formation of 
mRNA surveillance complexes containing hSMG-5 and hSMG-7, Mol Cell 12 (2003) $1187-1200$

[8] P. Nicholson, H. Yepiskoposyan, S. Metze, et al., Nonsense-mediated mRNA decay in human cells: mechanistic insights, functions beyond quality control and the double-life of NMD factors, Cell Mol Life Sci 67 (2010) 677-700.

[9] A. Yamashita, Role of SMG-1-mediated Upf1 phosphorylation in mammalian nonsense-mediated mRNA decay, Genes Cells 18 (2013) 161-175.

[10] M. Peccarelli, B.W. Kebaara, Regulation of natural mRNAs by the nonsense-mediated mRNA decay pathway, Eukaryot Cell 13 (2014) 1126-1135.

[11] C. Schweingruber, S.C. Rufener, D. Zund, et al., Nonsense-mediated mRNA decay mechanisms of substrate mRNA recognition and degradation in mammalian cells, Biochim Biophys Acta 1829 (2013) 612-623.

[12] G.C. Shukla, J. Singh, S. Barik, MicroRNAs: Processing, Maturation, Target Recognition and Regulatory Functions, Mol Cell Pharmacol 3 (2011) 83-92.

[13] D.P. Bartel, MicroRNAs: target recognition and regulatory functions, Cell 136 (2009) 215-233.

[14] I.G. Bruno, R. Karam, L. Huang, et al., Identification of a microRNA that activates gene expression by repressing nonsense-mediated RNA decay, Mol Cell 42 (2011) 500-510.

[15] A.E. Kulozik, Stay tuned: miRNA expression and nonsense-mediated decay in brain development, Mol Cell 42 (2011) 407-408.

[16] C.H. Lou, A. Shao, E.Y. Shum, et al., Posttranscriptional control of the stem cell and neurogenic programs by the nonsense-mediated RNA decay pathway, Cell Rep 6 (2014) 
748-764.

[17] G. Wang, B. Jiang, C. Jia, et al., MicroRNA 125 represses nonsense-mediated mRNA decay by regulating SMG1 expression, Biochem Biophys Res Commun 435 (2013) 16-20.

[18] J.T. Mendell, C.M. ap Rhys, H.C. Dietz, Separable roles for rent1/hUpf1 in altered splicing and decay of nonsense transcripts, Science 298 (2002) 419-422.

[19] F. Pastor, D. Kolonias, P.H. Giangrande, et al., Induction of tumour immunity by targeted inhibition of nonsense-mediated mRNA decay, Nature 465 (2010) 227-230.

[20] H. Le Hir, E. Izaurralde, L.E. Maquat, et al., The spliceosome deposits multiple proteins 20-24 nucleotides upstream of mRNA exon-exon junctions, EMBO J 19 (2000) $6860-6869$

[21] K.S. Rajavel, E.F. Neufeld, Nonsense-mediated decay of human HEXA mRNA, Mol Cell Biol 21 (2001) 5512-5519.

[22] K. Inoue, M. Khajavi, T. Ohyama, et al., Molecular mechanism for distinct neurological phenotypes conveyed by allelic truncating mutations, Nat Genet 36 (2004) 361-369.

[23] L.W. Harries, C. Bingham, C. Bellanne-Chantelot, et al., The position of premature termination codons in the hepatocyte nuclear factor -1 beta gene determines susceptibility to nonsense-mediated decay, Hum Genet 118 (2005) 214-224.

[24] J. Wang, J.P. Gudikote, O.R. Olivas, et al., Boundary-independent polar nonsense-mediated decay, EMBO Rep 3 (2002) 274-279.

[25] H.A. Kuzmiak, L.E. Maquat, Applying nonsense-mediated mRNA decay research to the clinic: progress and challenges, Trends Mol Med 12 (2006) 306-316.

[26] J.T. Mendell, H.C. Dietz, When the message goes awry: disease-producing mutations that 
influence mRNA content and performance, Cell 107 (2001) 411-414.

[27] R. Asselta, V. Rimoldi, I. Guella, et al., Molecular characterization of in-frame and out-of-frame alternative splicings in coagulation factor XI pre-mRNA, Blood 115 (2010) 2065-2072.

[28] M.D. Bashyam, Studies on nonsense mediated decay reveal novel therapeutic options for genetic diseases, Recent Pat DNA Gene Seq 3 (2009) 7-15.

[29] A.S. Ramalho, S. Beck, M. Meyer, et al., Five percent of normal cystic fibrosis transmembrane conductance regulator mRNA ameliorates the severity of pulmonary disease in cystic fibrosis, Am J Respir Cell Mol Biol 27 (2002) 619-627.

[30] E.M. Welch, E.R. Barton, J. Zhuo, et al., PTC124 targets genetic disorders caused by nonsense mutations, Nature 447 (2007) 87-91.

[31] S. Hirawat, E.M. Welch, G.L. Elfring, et al., Safety, tolerability, and pharmacokinetics of PTC124, a nonaminoglycoside nonsense mutation suppressor, following single- and multiple-dose administration to healthy male and female adult volunteers, J Clin Pharmacol 47 (2007) 430-444. 
Table 1

Table 1. Oligonucleotides used in this study

\begin{tabular}{cc}
\hline Oligonucleotides & Sequence 5'-3' \\
\hline Target UPF1 & AAGATGCAGTTCCGCTCCATTTT \\
Target SMG1 & GCCACCAAAGACATGAGGAAA \\
Target Control & AATTCTCCGAACGTGTCACTT \\
BF319 & ATTAGCGAGGAAGACCTCGGATCC \\
BF320 & CCGGCACATCATAGGGATAAAGCTT \\
BF321 & GTTAAAATTACAGTTGTCGCAGGTGAA \\
BF324 & TCCAGTTCCAGAAGGGCAATGT \\
BF325 & ATTCGACCACCAAGCGAAACA \\
BF326 & CCTTGAGCCTGGCGAACAGTT \\
\hline
\end{tabular}


Figure 1

A

\begin{tabular}{|l|l|}
\hline $6 \times$ myc & Exon 1 \\
\hline
\end{tabular}



B

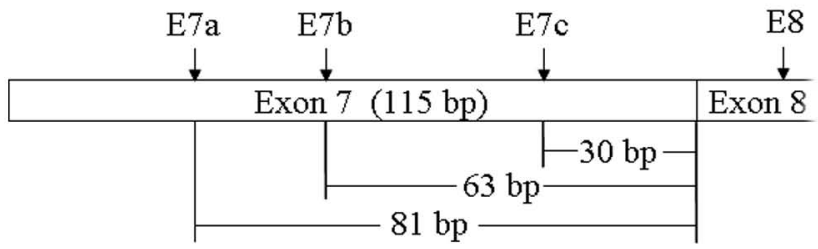

C

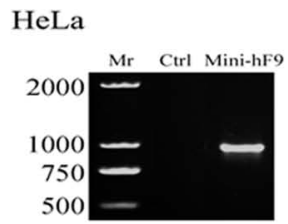

\section{COS7}

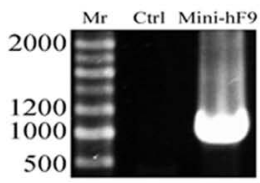

HEK 293T

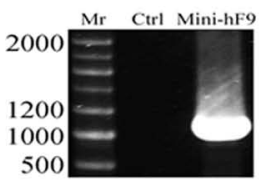

HepG2

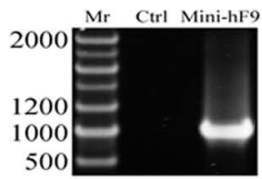

D



Anti-HA
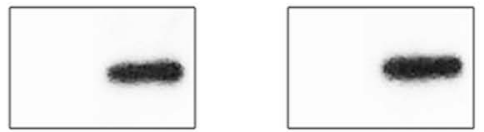
A Figure 2

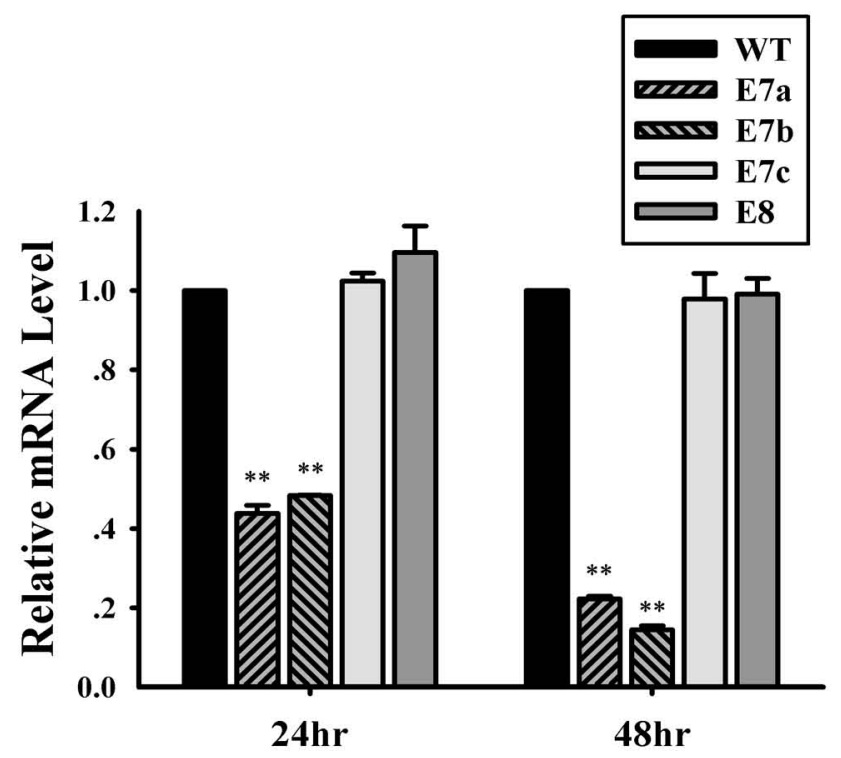

C

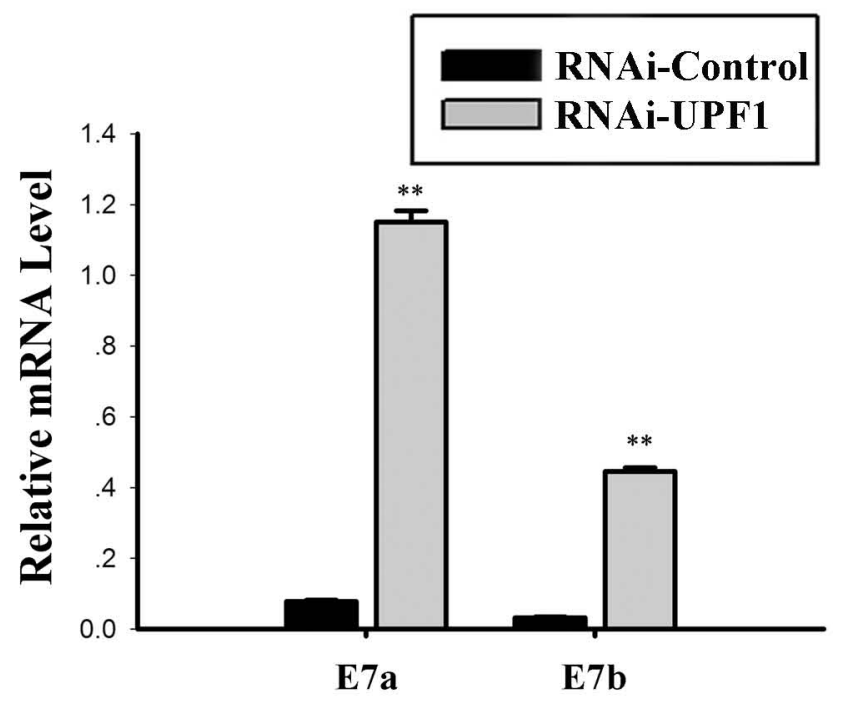

$\mathbf{E}$

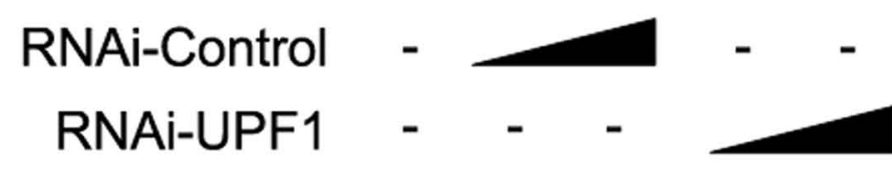

Anti-hUPF1

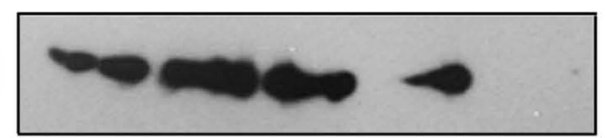

Anti- $\beta$-actin
B

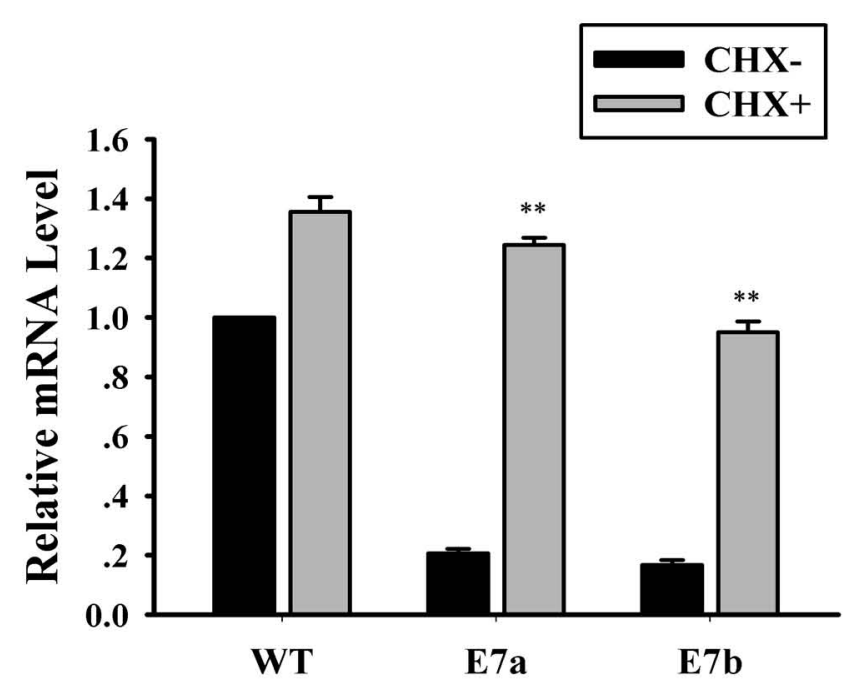

D

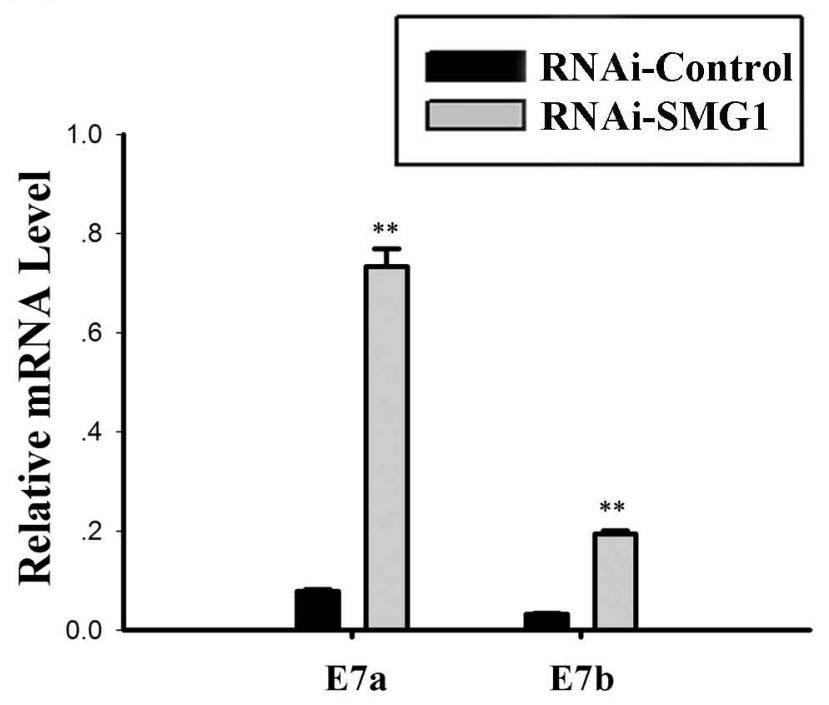

F

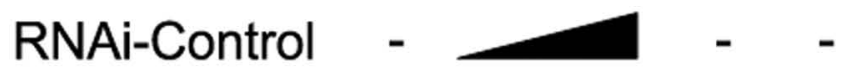
RNAi-SMG1 - - -

Anti-hSMG1

Anti- $\beta$-actin 
Figure 3

\section{WT E7a E7b E7c E8}

Anti-c-myc

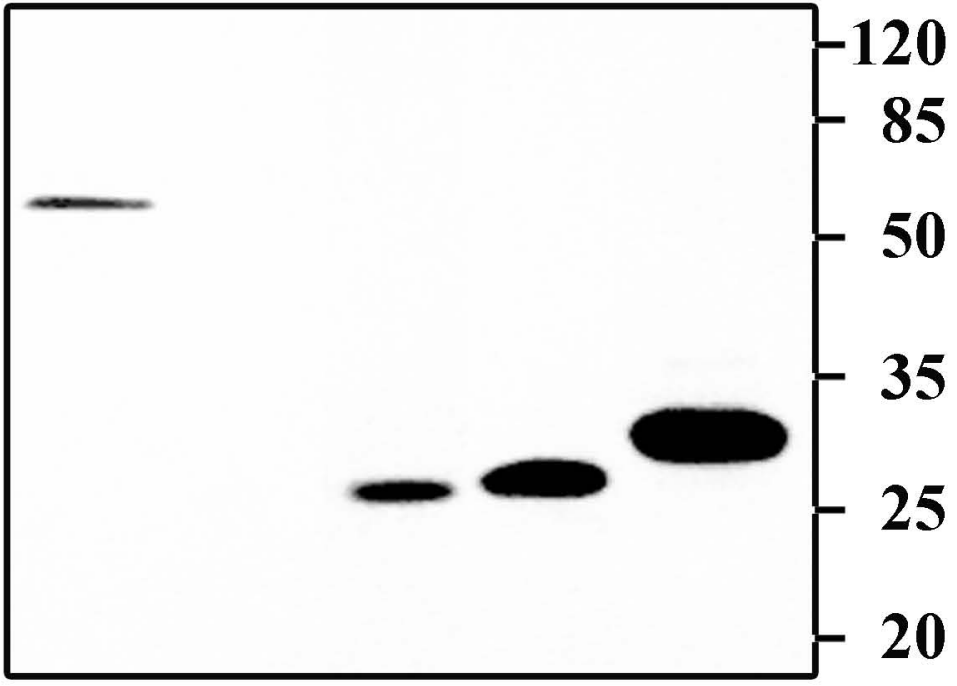

Anti- $\beta$-actin

$\begin{array}{lllll}1.0 & 0.0 & 1.2 & 3.2 & 4.1\end{array}$

B

E7a E7b E7c E8

Anti-c-myc





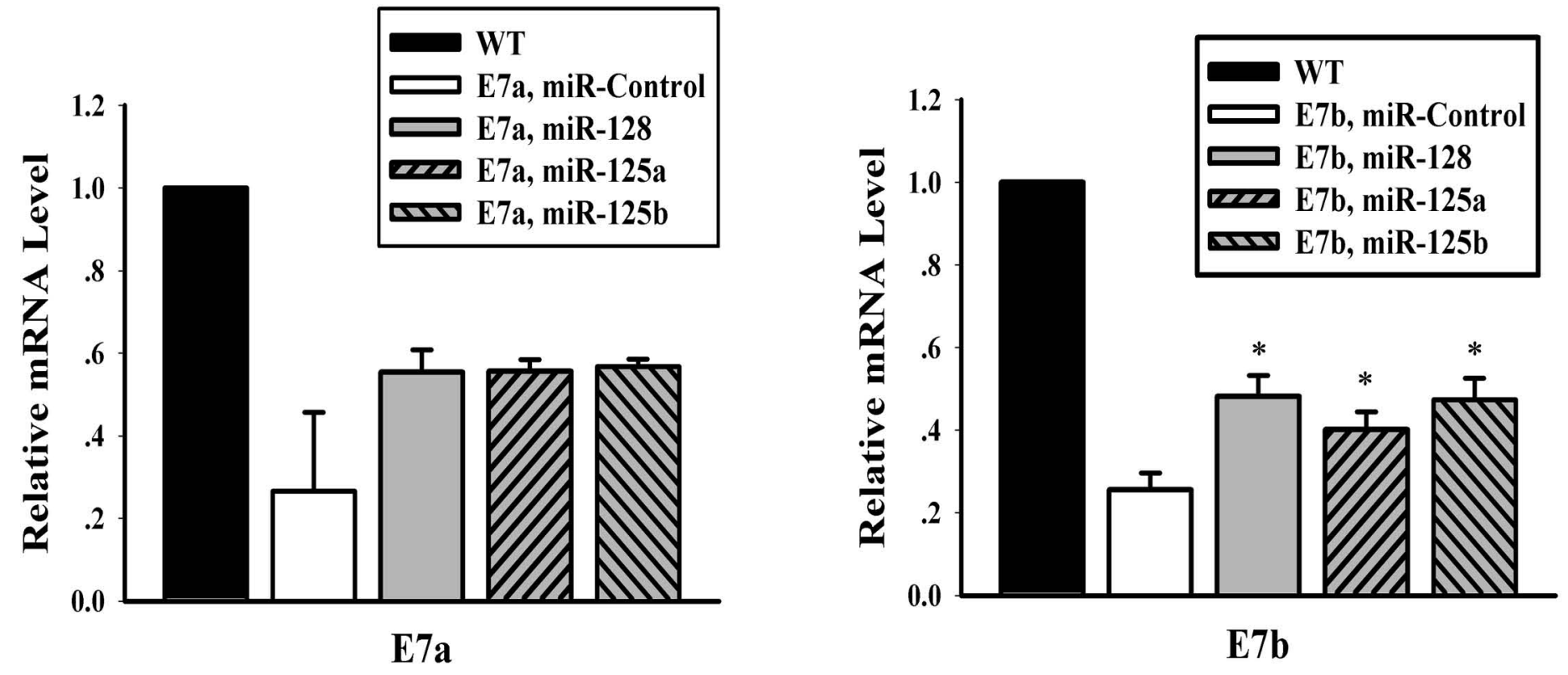